\title{
Synthesis of 5-aryl-4-acyl-3-hydroxy- 1-carboxymethyl-3-pyrrolin-2-ones
}

\author{
(C) Vladimir L. Gein, ${ }^{+}$and Evgenia V. Pastukhova \\ Department of General and Organic Chemistry. Perm State Academy of Pharmacy. \\ Polevaya St., 2.Perm, 614090. Russia.Phone: +7 (342) 236-90-50.E-mail: geinvl48@mail.ru
}

*Supervising author; ${ }^{+}$Corresponding author Keywords: 5-aryl-4-acyl-3-hydroxy-1-carboxymethyl-3-pyrrolin-2-ones, glycocol, tetrahydropyrrole-2,3-diones, three-component reactions.

\begin{abstract}
5-Aryl-1,4-disubstituted terahydropyrrole-2,3-diones are five-membered nitrogen heterocycles containing in the first alkyl position or an aryl functionalized substituent and in the fourth position in another state of nature an acyl residue. Trahydropyrrole-2,3-diones constitute a significant class of available and stable substances. Along with this, they easily enter into reactions with various nucleophilic reagents due to the highly reactive carbonyl group in the third position of the heterocycle. The presence of the latter, as well as the carbonyl group of the side chain, makes it possible to form various condensed systems from heterocycles in reactions with binucleophilic reagents. The synthesis of biologically active substances based on 1,4,5trisubstituted tetrahydropyrrole-2,3-diones is one of the promising ways of using this class of compounds. Previously, it was found that 1,4,5-trisubstituted 3-hydroxy-3-pyrin-2-ones have anti-inflammatory, analgesic, antimicrobial, nootropic, antiplatelet and antiviral activity.

We set the task to obtain 5-aryl-4-acyl-3-hydroxy-3-pyrrolin-2-ones, in the first position of the heterocycle there is a carboxymethyl substituent. By setting up a three-component reaction of acylpyruvic acid ether with aromatic aldehydes and glycine in dioxane in dioxane. The structure of the compound was established on the basis of IR and ${ }^{1} \mathrm{H}$ NMR spectroscopy data. Based on the data of ${ }^{1} \mathrm{H}$ NMR spectroscopy and a positive reaction with an alcoholic solution of iron(III) chloride, the predominant presence of compounds in the enol form was established.

The article presents the proposed mechanism and schemes of the formula for the synthesis of 5-aryl-4acyl-3-hydroxy-1-carboxymethyl-3-pyrrolin-2-ones, their physicochemical and spectral characteristics, as well as the yields of the reaction products.
\end{abstract}

\section{References}

[1] V.L Gein. Tetrahydropyrrole- and tetrahydrofuran-2,3-diones. Perm State Pharmaceutical Academy, Perm. 2004. P.130. (russian)

[2] M.A. Maryasov, V.L. Gein. Tetrahydropyrrole-2,3-diones: monograph. Perm: Perm State Pharmaceutical Academy. 2013. P.155. (russian)

[3] L.F. Gein. Synthesis, chemical properties and biological activity of 1,4-disubstituted 5-aryl-3-hydroxy-3pyrrolin-2-ones: abstract of the dissertation of Doctor of Pharmaceutical Sciences. 2009. P.51. (russian)

[4] I.A. Kylosova. Synthesis, properties and biological activity of 5-aryl-4-acyl-3-hydroxy-1-carboxyalkyl-3pyrrolin-2-ones and their functional derivatives: Abstract of Ph.D. Thesis. Perm. 2007. P.21. (russian) 